Series A

I. MATHEMATICA

586

\title{
LINEAR EXTREMAL PROBLEMS FOR ANALYTIC FUNCTIONS WITH INTERIOR SIDE CONDITIONS
}

BY

DENNIS A. HEJHAL

HEL S I K I 1974

S U O M A L A I N E T I E D A K A T EMIA

doi:10.5186/aasfm.1975.586 


\author{
Copyright (C) 1974 by \\ Academia Scientiarum Fennica \\ ISSN 0066-1953 \\ ISBN 951-41-0200-2
}

Communicated 11 February 1974 


\section{Contents}

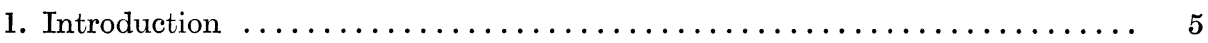

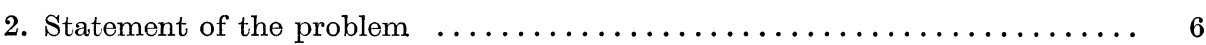

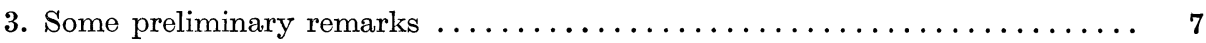

4. Ordinary linear extremal problems $\ldots \ldots \ldots \ldots \ldots \ldots \ldots \ldots \ldots \ldots . . \ldots \ldots$

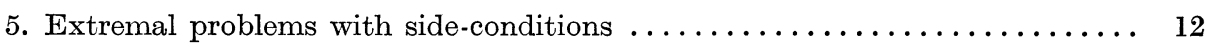

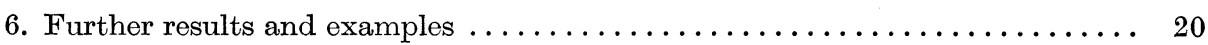

7. Further discussion of Carleman-Milloux problems $\ldots \ldots \ldots \ldots \ldots \ldots \ldots 24$

8. Further discussion of Pick-Nevanlinna problems ................ 29

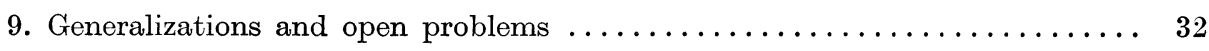




\section{Introduction}

In this paper we propose to show how the method of dual extremal problems can be applied to problems involving analytic functions which satisfy interior side-conditions. The extremal problems we study have their origins in the classical problems of Carleman-Milloux and PickNevanlinna.

The Carleman-Milloux problem for analytic functions is concerned with the family

$$
\mathcal{C}=\{f \in A(U):|f(z)| \leqq 1 \text { on } U,|f(z)| \leqq \delta \text { on } E\} .
$$

Here $U$ denotes the unit disk, $\delta$ is a positive constant $0<\delta<1$, and $E$ is a path which runs from 0 to 1 , say. Let $z_{0} \in U-E$. The set $\left\{f\left(z_{0}\right): f \in^{\mathcal{C}}\right\}$ will then be a closed disk $|w| \leqq M$. Functions $F \in \mathscr{C}$ which satisfy $\left|F\left(z_{0}\right)\right|=M$ are called extremal functions. The problem is to describe the extremal functions $F$ and to calculate $M$.

A potential-theoretic approach to this problem can be found in [20, p. 112]. However, since the extremal functions obtained in this way are not single-valued, this approach gives only partial results. The complete solution was given by Heins [13] in 1945 .

The Pick-Nevanlinna interpolation problem, on the other hand, is concerned with the family

$$
\mathcal{C}=\left\{f \in A(U):|f(z)| \leqq 1 \text { on } U, f\left(\xi_{1}\right)=a_{1}, \ldots, f\left(\xi_{m}\right)=a_{m}\right\},
$$

where $\xi_{1}, \ldots, \xi_{m}$ are distinct points in $U$ and the $a_{k}$ are complex numbers. We assume that $z_{0} \in U-\left\{\xi_{1}, \ldots, \xi_{m}\right\}$ and let $W=\left\{f\left(z_{0}\right): f \in \mathcal{C}\right\}$. The set $W$, which may well be empty, is closed and convex. The extremal functions $F \in \mathcal{C}$ are those which satisfy $F\left(z_{0}\right) \in \partial W$. One would like to describe, for example, those extremal functions which satisfy $\operatorname{Re} F\left(z_{0}\right)=$ $M=$ maximum . The classical treatment of this problem can be found in [21] and [24, pp. 281-309].

If one tries to extend these classical developments to multiply-connected domains, difficulties soon appear and it quickly becomes apparent that

Some abbreviations: $\mathbf{C}=$ complex plane, $\mathbf{R}=$ real line, iff $=$ if and only if, wlog $=$ without loss of generality. 
new ideas are called for. In the case of the Pick-Nevanlinna problem, we may refer to [7, pp. 25-32] and [12].

Now, as is well-known, many extremal problems on multiply-connected domains can be formulated as dual extremal problems. This was first proved by Garabedian [7] for the Schwarz lemma and has since been the subject of numerous papers (e.g. [15]).

The obvious question is thus whether problems with interior sideconditions can be formulated as dual extremal problems. Very little was known about this until around 1963, when Havinson [9] found a dual extremal problem for the general Carleman-Milloux problem. Very recently, Gamelin [6] showed how the general Pick-Nevanlinna problem can be transformed into a dual extremal problem. Both of these developments require a certain amount of abstract functional analysis.

The method we shall explain here is applicable to quite general linear extremal problems with interior side-conditions and is, moreover, entirely classical in nature. Our work therefore both complements and extends results found in [6] and [9].

It will be seen that our method consists of essentially three parts: (a) the study of the minimum problem by variational methods; (b) reduction to simpler extremal problems; and (c) approximation.

For purposes of illustration, it will suffice to work in a situation of moderate generality. The techniques we use apply much more generally: some of the possible generalizations are indicated at the end, in section 9 .

Finally, it is a pleasure for me to thank Professors L. Ahlfors, H. Royden, and M. Schiffer for a number of very interesting discussions about extremal problems. Most of the work described in this paper was done at Stanford University.

\section{Statement of the problem}

We begin with the following list of assumptions:

(i) $\quad D$ is a plane domain with analytic boundary $\partial D$ and connectivity $p, \quad 1 \leqq p<\infty$;

(ii) $E=E_{1} \cup \ldots \cup E_{m}$, where the $E_{k}$ are mutually disjoint compact subsets of $D$;

(iii) $K$ is a compact subset of $D$;

(iv) $\lambda$ is a totally finite complex Borel measure on $K$;

(v) $\mathscr{L}[h]=\int_{\boldsymbol{K}} h d \lambda$ for $h \in C(K)$; 
(vi) $k(z)$ is the Cauchy transform

$$
k(z)=\frac{1}{2 \pi i} \int_{K} \frac{1}{z-t} d \lambda(t) ;
$$

(vii) $a_{k} \in \mathbf{C}, \quad \delta_{k} \geqq 0$ for $1 \leqq k \leqq m$;

(viii) each component of $\mathbf{C}-E$ intersects $\partial D$;

(ix) we write

$$
\ell=\left\{f \in A(D):|f(z)| \leqq 1 \text { on } D,\left|f(z)-a_{k}\right| \leqq \delta_{k} \text { on } E_{k}\right\} .
$$

A few words regarding the notation: (a) an analytic Jordan curve necessarily admits a parametric representation $\xi=\xi(x), 0 \leqq x \leqq 1$, in which $\xi(t)$ is analytic, has period 1 , and is schlicht $(\bmod 1)$ on some strip $|\operatorname{Im}(t)|<\eta$; (b) $A(D)$ denotes the family of single-valued analytic functions on $D$; (c) $C(K)$ denotes the family of continuous functions on $K$.

We might also mention that condition (viii) ensures that the various conditions $\left|f(z)-a_{k}\right| \leqq \delta_{k}$ do not interfere with each other (under the maximum modulus principle).

Fundamental problem. Assume that $\ell$ is non-void and let $M=$ $\sup \operatorname{Re} \mathscr{L}(f)$ over all $f \in \mathscr{C}$. We want to describe the extremal functions $F \in \ell$ which satisfy $\operatorname{Re} \mathscr{L}(F)=M$.

Of course, since $C$ is a normal family, the existence of such $F \in C^{\top}$ is guaranteed.

By choosing (a) $\quad a_{k}=0, \quad \delta_{k}=\delta$ and (b) $\delta_{k}=0, E_{k}=\left\{\xi_{k}\right\}$ we obtain extremal problems of Carleman-Milloux and Pick-Nevanlinna type, respectively. The case (c) $a_{k}=0, \delta_{k}=0, E_{k}=\left\{\xi_{k}\right\}$ is much like the Schwarz lemma and will be called an ordinary linear extremal problem (see [15]).

\section{Some preliminary remarks}

In our development, we shall make implicit use of the non-tangential boundary values of analytic functions of class $A B(D)$ and $H_{1}(D)$. The classes $A B(D)$ and $H_{1}(D)$ are defined as follows:

$$
\begin{gathered}
A B(D)=\{f \in A(D): \mathrm{f} \text { is bounded on } D\} ; \\
H_{1}(D)=\{f \in A(D):|f| \text { has a harmonic majorant on } D\} .
\end{gathered}
$$

When $D=U, H_{1}(D)$ reduces to the well-known Hardy $H_{1}$ class; in this regard, see also [14]. The properties we use are classical for $p=1$ and straightforward extensions when $2 \leqq p<\infty$. We may refer to [8], 
[10], and [22]. Note too that, since $D$ was assumed to be analytic, $H_{1}(D)$ coincides with the Smirnow class $E_{1}(D)$. Lastly, we shall frequently use the common notation $\|f\|=\sup |f(z)|$, when $f \in A B(D)$.

The following three results will prove particularly useful. It is convenient to let $D_{\varepsilon}$ be the subdomain of $D$ which is bounded by the curves $\xi=\xi(x+i \varepsilon), \varepsilon>0$.

Lemma 1. There exists a positive function $C(\varepsilon)$ such that $C(\varepsilon) \rightarrow 1$ as $\varepsilon \rightarrow 0$ and

$$
\int_{\partial D_{\varepsilon}}|g(z)||d z| \leqq C(\varepsilon) \int_{\partial D}|g(\xi)||d \xi|
$$

for all $g \in H_{1}(D)$.

Proof (sketch). For $z=\xi(x+i \varepsilon) \in \partial D_{\varepsilon}$, we define the reflection $z^{*}=\xi(x-i \varepsilon)$. Then, for $g \in H_{1}(D)$,

$$
\begin{gathered}
g(z)=\frac{1}{2 \pi i} \int_{\partial D} g(t)\left[\frac{1}{t-z}-\frac{1}{t-z^{*}}\right] d t \\
\int_{\partial D_{\varepsilon}}|g(z)||d z| \leqq \frac{1}{2 \pi} \int_{\partial D}|g(t)|\left[\int_{\partial D_{\varepsilon}}\left|\frac{1}{z-t}-\frac{1}{z^{*}-t}\right||d z|\right]|d t| .
\end{gathered}
$$

We claim that

$$
C(\varepsilon)=\sup _{t \in \partial D} \frac{1}{2 \pi} \int_{\partial D_{\varepsilon}}\left|\frac{1}{z-t}-\frac{1}{z^{*}-t}\right||d z|
$$

does the job. In fact, we need only check that $\lim _{\varepsilon \rightarrow 0} C(\varepsilon)=1$. But this is easy, since $\partial D$ is analytic and the integral behaves like a Poisson integral.

Lemma 2. Let $f_{n}(z)$ be a pointwise convergent sequence of $H_{1}(D)$ functions whose limit function is $f(z)$. Suppose further that the boundary integrals $\int_{\partial D}\left|f_{n}(\xi)\right||d \xi|$ remain bounded. Then, $f(z) \in H_{1}(D)$ and

$$
\int_{\partial D}|f(\xi)||d \xi| \leqq \liminf _{n \rightarrow \infty} \int_{\partial D}\left|f_{n}(\xi)\right||d \xi| .
$$

Proof(sketch). By the Cauchy integral formula, the $f_{n}(z)$ are uniformly bounded on $D$ compacta. It follows that $f(z) \in A(D)$ and that the convergence is uniform on every $D_{\varepsilon}$. By Lemma 1 , 


$$
\int_{\partial D_{\varepsilon}}|f(z)||d z|=\lim _{n \rightarrow \infty} \int_{\partial D_{\varepsilon}}\left|f_{n}(z)\right||d z| \leqq C(\varepsilon) \liminf _{n \rightarrow \infty} \int_{\partial D}\left|f_{n}(\xi)\right||d \xi|
$$

for each $\varepsilon>0$. The lemma follows at once, since $H_{1}(D)=E_{1}(D)$.

Lemma 3. Let $h(\xi)$ belong to $L_{1}(\partial D)$ or $L_{\infty}(\partial D)$, respectively. A necessary and sufficient condition for $h(\xi)$ to coincide with the boundary value of a function in $H_{1}(D)$ or $A B(D)$, respectively, is that

$$
\int_{\partial D} f(\xi) h(\xi) d \xi=0
$$

for every $f \in A(D \cup \partial D)$.

Proof(sketch). The necessity is clear. To prove the sufficiency, we simply study

$$
H(z)=\frac{1}{2 \pi i} \int_{\partial D} \frac{h(\xi)}{\xi-z} d \xi .
$$

For $z \in \partial D_{\varepsilon}$, clearly $H\left(z^{*}\right)=0$. Therefore, for $z$ near $\partial D$,

$$
H(z)=-\frac{1}{2 \pi i} \int_{\partial D} h(\xi)\left[\frac{1}{\xi-z}-\frac{1}{\xi-z^{*}}\right] d \xi .
$$

Since $\partial D$ is analytic, this integral behaves like a Poisson integral and

$$
\int_{\partial D_{\varepsilon}}|H(z)||d z| \leqq C(\varepsilon) \int_{\partial D}|h(\xi)||d \xi|
$$

as in the proof of Lemma 1. See also [22, pp. 144-145].

\section{Ordinary linear extremal problems}

According to the definition in section 2, we now have

$$
\mathcal{e}=\left\{f \in A(D):|f(z)| \leqq 1 \text { on } D, f\left(\xi_{1}\right)=\ldots=f\left(\xi_{m}\right)=0\right\}
$$

and $M=\sup \operatorname{Re} \mathscr{L}(f)=\sup |\mathscr{L}(f)|$ for $f \in \mathscr{C}$. To see how the dual extremal problem arises in this case, we observe that

$$
\begin{gathered}
\operatorname{Re} \mathscr{L}(f)=\operatorname{Re} \int_{\partial D} f(\xi) k(\xi) d \xi=\operatorname{Re} \int_{\partial D} f(\xi)[k-\omega-\varphi] d \xi \\
\leqq \int_{\partial D}|k-\omega-\varphi||d \xi|
\end{gathered}
$$


where $f \in \ell, \varphi \in H_{1}(D)$, and $\omega$ has the form $(2 \pi i)^{-1} \sum_{k=1}^{m} \mu_{k}\left(z-\xi_{k}\right)^{-1}$, $\mu_{k} \in \mathbf{C}$. Therefore,

$$
M \leqq M_{0}=\inf _{\omega, \varphi} \int_{\partial D}|k-\omega-\varphi||d \xi| .
$$

The following well-known result then holds.

Theorem 1. Assume that $M \neq 0$. Then:

(i) $\quad M=M_{0}$ and $M_{0}$ is actually a minimum, that is, $M_{0}$ is assumed for some pair $\left(\omega_{0}, \varphi_{0}\right)$;

(ii) the extremal function $F \in \mathscr{C}$ is unique: $\mathscr{L}(F)=M$;

(iii) $\varphi_{0}(z)$ remains analytic across $\partial D$;

(iv) $F(z)\left(k-\omega_{0}-\varphi_{0}\right)$ is analytic across $\partial D, F\left(k-\omega_{0}-\varphi_{0}\right) d z \geqq 0$ on $\partial D$;

(v) for each component $\Gamma$ of $\partial D$, there are exactly two possibilities: (1) $k \neq \varphi_{0}+\omega_{0}$ near $\Gamma, F$ analytic across $\partial D,|F|=1$ on $\Gamma$;

(2) $k \equiv \varphi_{0}+\omega_{0}$ near $\Gamma$, with nothing asserted about $F$.

Proof. A proof of this result can be found in [15, pp. 94-99] or [19]. These proofs employ the Hahn-Banach theorem, however. To avoid this, one can proceed as follows.

It will suffice to prove that $M=M_{0}$, since the rest then follows in an entirely classical fashion. See [15, pp. 96-99] and Lemmas 1, 2.

Suppose first that $K$ is a finite set. Let us write

$$
I[\omega, \varphi]=\int_{\partial D}|k-\omega-\varphi||d \xi|, \quad I\left[\omega_{0}, \varphi_{0}\right]=M_{0},
$$

and $A=k-\omega_{0}-\varphi_{0}$. Using $M_{0} \geqq M>0$, the fact that $k(z)$ is now a rational function, and the Lusin-Riesz-Priwalow theorem [22, p. 212], we can assume wlog that $A(\xi) \neq 0, \infty$ everywhere on $\partial D$. Define $\quad U_{r}=\{\xi \in \partial D:|A(\xi)|>r\}, \quad V_{r}=\{\xi \in \partial D: \mid A(\xi) \leqq r\}$, and $T(r)=\left[r+m\left(V_{r}\right)\right] r$ for $r>0$. Of course, by measure theory,

$$
\lim _{r \rightarrow 0} m\left(V_{r}\right)=0 \text {. }
$$

Choose any $h \in A(D \cup \partial D)$ and consider complex numbers $t$ such that $|t|=T(r)$. We want to analyze the condition

$$
I\left[\omega_{0}, \varphi_{0}+t h\right]-I\left[\omega_{0}, \varphi_{0}\right] \geqq 0
$$


as $r \rightarrow 0$. Using the obvious inequality $|A|-|t h| \leqq|A-t h| \leqq|A|+|t h|$, we readily check that

$$
\int_{U_{r}}|A|\left[\left|1-\frac{t h}{A}\right|-1\right]|d \xi|+O\left[T(r) m\left(V_{r}\right)\right] \geqq 0 .
$$

On $U_{r}$, however,

$$
\left|\frac{t}{A}\right|=\frac{T(r)}{|A|} \leqq \frac{T(r)}{r} \rightarrow 0,
$$

so that

$$
\left|1-\frac{t h}{A}\right|=1-\operatorname{Re}\left(\frac{t h}{A}\right)+O\left(\left|\frac{t}{A}\right|^{2}\right) .
$$

Substitution of this estimate yields

$$
O\left[T(r) m\left(V_{r}\right)\right]-\operatorname{Re}\left[t \int_{U_{r}} h \frac{|A|}{A}|d \xi|\right]+O\left[\frac{T(r)^{2}}{r}\right] \geqq 0 .
$$

Upon writing $t=T(r) e^{i \ominus}$, we find that

$$
O\left[m\left(V_{r}\right)\right]-\operatorname{Re}\left[e^{i \theta} \int_{U_{\boldsymbol{r}}} h \frac{|A|}{A}|d \xi|\right]+O\left[\frac{T(r)}{r}\right] \geqq 0,
$$

for each value of $\theta$ as $r \rightarrow 0$. It follows then that

$$
\int_{\partial D} h(\xi) \frac{|A|}{A}|d \xi|=0, h \in A(D \cup \partial D) .
$$

By Lemma 3 and its proof, we deduce that

$$
F(\xi) A(\xi) d \xi=|A(\xi)||d \xi| \text { a.e. }
$$

for some $F \in A B(D),|F(z)| \leqq 1$. Moreover, if we repeat the above argument with $h(z)$ replaced by $\left(z-\xi_{k}\right)^{-1}$, we immediately see that

$$
0=\int_{\partial D} \frac{1}{\xi-\xi_{k}} \frac{|A|}{A}|d \xi|=\int_{\partial D} \frac{F(\xi)}{\xi-\xi_{k}} d \xi
$$

whence $F\left(\xi_{k}\right)=0$. That is, $F \in \mathcal{C}$. By construction, $\mathscr{L}(F)=M_{0}$. Thus $M=M_{0}$, as required.

To prove $M=M_{0}$ in the general case, we shall use approximation. We fix any small $\eta>0$ and let $C=\partial D_{\eta}$. Then, $\mathscr{L}[f]=\int_{C} f(z) k(z) d z$ for all $f \in A B(D)$. We next partition $C$ into $N$ small pieces $C_{\alpha}$ so 
that, on each piece, the total variation of any $f \in A B(D)$ is $\leqq \varepsilon\|f\|$. Choose points $z_{\alpha} \in C_{\alpha}, 1 \leqq \alpha \leqq N$, and define a discrete measure $v$ by means of

$$
v\left\{z_{\alpha}\right\}=\int_{c_{\alpha}} k(t) d t .
$$

In an obvious notation, let $\mathscr{L}_{\varepsilon}$ be the linear functional represented by $\boldsymbol{v}_{\varepsilon}$.

We have already proved that Theorem 1 holds whenever $K$ is finite. We may therefore determine dual extremal data $F_{\varepsilon}, k_{\varepsilon}, \omega_{\varepsilon}, \varphi_{\varepsilon}, M_{\varepsilon}$ for $\mathscr{L}_{\varepsilon}$ over $\ell$. It is important to observe here that

$$
\left|\mathscr{L}_{\varepsilon}(f)-\mathscr{L}(f)\right| \leqq \varepsilon\|f\| \int_{C}|k(t)||d t|, \quad f \in A B(D) .
$$

A simple normal families argument then shows that $M_{\varepsilon} \rightarrow M$ as $\varepsilon \rightarrow 0$. Since $M_{\varepsilon}=\int_{\partial D}\left|k_{\varepsilon}-\omega_{\varepsilon}-\varphi_{\varepsilon}\right||d z|$, it follows that

$$
M_{0} \leqq M_{\varepsilon}+\int_{\partial D}\left|k_{\varepsilon}-k\right||d z| .
$$

However, it is easily checked that $k_{\varepsilon}(z) \rightarrow k(z)$ along $\partial D$. Therefore $M_{0} \leqq M$ and the proof is complete.

Remark 1. It should be noted that in (iii)-(v) any minimizing pair $\left(\varphi_{0}, \omega_{0}\right)$ can be used. We also observe that since $M=M_{0} \neq 0$, it follows that $k \neq \varphi_{0}+\omega_{0}$, so that possibility (1) in item (v) must hold at least once.

Remark 2. The proof given above for Theorem 1 was motivated in part by Carleson [3, pp. 78-82].

\section{Extremal problems with side-conditions}

We now consider the general problem posed in section 2. To discover the appropriate dual extremal problem, we observe that:

$$
\begin{gathered}
\operatorname{Re} \mathscr{L}(f)=\operatorname{Re} \int_{\partial D} f(z) k(z) d z=\operatorname{Re} \int_{\partial D} f\left(k-\omega_{\mu}-\varphi\right) d z+\operatorname{Re} \int_{E} f d \mu \\
\leqq \int_{\partial D}\left|k-\omega_{\mu}-\varphi\right||d z|+\operatorname{Re}\left\{a_{\alpha} \mu\left(E_{\alpha}\right)\right\}+\delta_{\alpha}|\mu|\left(E_{\alpha}\right)
\end{gathered}
$$


for each $f \in \mathscr{C}$, where $\mu$ is a totally finite complex Borel measure on $E, \varphi \in H_{1}(D)$, and $\omega_{\mu}(z)$ denotes the Cauchy transform

$$
\omega_{\mu}(z)=\frac{1}{2 \pi i} \int_{E} \frac{1}{z-t} d \mu(t) \text {. }
$$

Note too that we use the Einstein summation convention over the repeated indices $\alpha$. It follows then that

$$
M \leqq M_{0}=\inf _{\mu, \varphi}\left[\int_{\partial D}\left|k-\omega_{\mu}-\varphi\right||d z|+\operatorname{Re}\left\{a_{\alpha} \mu\left(E_{\alpha}\right)\right\}+\delta_{\alpha}|\mu|\left(E_{\alpha}\right)\right] .
$$

These inequalities will lead to a dual extremal problem provided that $M=M_{0}$ and that $M_{0}$ is actually assumed for some pair $(\eta, \Phi)$. In that case, one can clearly start reading off properties of the extremal functions $F \in \mathscr{C}$. For example, $F\left(k-\omega_{\eta}-\Phi\right) d z=\left|k-\omega_{\eta}-\Phi\right||d z|$ along $\partial D$.

We intend to prove the following two fundamental theorems in this section. In stating them, we shall call $C$ non-trivial iff cardinal $(e) \geqq 2$. Similarly, $e$ is called trivial iff cardinal $(e)=1$.

Theorem 2. Assume that $\circlearrowright$ is non-trivial. Every minimizing sequence $\left(\mu_{n}, \varphi_{n}\right)$ for problem $M_{0}$ is then bounded. That is, $\left|\mu_{n}\right|(E)$ and $\int_{\partial D}\left|\varphi_{n}(z)\right||d z|$ remain bounded when $n \rightarrow \infty$.

Theorem 3. If $e$ is non-trivial, then $M=M_{0}$ and $M_{0}$ is actually a minimum. When $C$ is trivial, $M=M_{0}$ still holds, but $M_{0}$ need not be a minimum.

Proof (Theorem 2). Let $\left(\mu_{n}, \varphi_{n}\right)$ be a minimizing sequence for problem $M_{0}$. Suppose that $\left\|\mu_{n}\right\|=\left|\mu_{n}\right|(E) \rightarrow \infty$ as $n \rightarrow \infty$. Then,

$$
\begin{gathered}
\int_{\partial D} \frac{k}{\mu_{n} \|}-\frac{\omega_{\mu_{n}}}{\left\|\mu_{n}\right\|}-\frac{\varphi_{n}}{\left\|\mu_{n}\right\|}|d z|+\operatorname{Re}\left\{a_{\alpha} \frac{\mu_{n}\left(E_{\alpha}\right)}{\left\|\mu_{n}\right\|}\right\} \\
+\delta_{\alpha} \frac{\left|\mu_{n}\right|\left(E_{\alpha}\right)}{\left\|\mu_{n}\right\|} \rightarrow 0
\end{gathered}
$$

We now apply the selection theorem to the totally bounded measures $\mu_{n}\left\|\mu_{n}\right\|^{-1}$ on $E$. Therefore, wlog,

$$
\frac{\mu_{n}}{\left\|\mu_{n}\right\|} \stackrel{w^{*}}{\rightarrow} \eta
$$

where $\eta$ is a totally finite complex Borel measure on $E$. Furthermore, wlog, 


$$
\frac{\left|\mu_{n}\right|}{\left\|\mu_{n}\right\|} \stackrel{w^{*}}{\rightarrow} Q
$$

where $Q$ is a probability measure on $E$. It follows that

$$
|\eta| \leqq Q
$$

Note: The notation $i_{n} \stackrel{w^{*}}{\rightarrow} i$ is used to denote weak-star convergence, which is to say that

$$
\int_{E} f d i_{n} \rightarrow \int_{E} f d i
$$

for every $f \in C(E)$. To prove the assertion $|\eta| \leqq Q$, one recalls the definition of $|\eta|$ as in [18, pp. 308-309] and first checks that $|\eta|(F) \leqq Q(F)$ for compact sets $F$.

By the $w^{*}$ convergence, clearly

$$
\frac{\omega_{\mu_{n}}}{\left\|\mu_{n}\right\|} \rightarrow \omega{ }_{\eta}
$$

near $\partial D$. By a normal families argument and Lemma 2, wlog

$$
\frac{\varphi_{n}(z)}{\left\|\mu_{n}\right\|} \rightarrow \varphi_{\infty}(z)
$$

on $D$ compacta, $\varphi_{s} \in H_{1}(D)$. Moreover, by means of a simple extension of Lemma 2, we see that

$$
\int_{\partial \boldsymbol{D}}\left|-\omega_{\eta}-\varphi_{\infty}\right||d z|+\operatorname{Re}\left\{a_{\alpha} \eta\left(E_{\alpha}\right)\right\}+\delta_{\alpha} Q\left(E_{\alpha}\right) \leqq 0 .
$$

Hence,

$$
\int_{\partial D}\left|-\omega_{\eta}-\varphi_{\infty}\right||d z|+\operatorname{Re}\left\{a_{\alpha} \eta\left(E_{\alpha}\right)\right\}+\delta_{\alpha}|\eta|\left(E_{\alpha}\right) \leqq 0 .
$$

We claim that $\| \eta^{\prime \prime}=|\eta|(E) \neq 0$. If not, then

$$
\int_{\partial D}\left|\varphi_{\infty}\right||d z|+\delta_{\alpha} Q\left(E_{\alpha}\right) \leqq 0 \text {. }
$$

Hence, $\quad \delta_{\alpha} Q\left(E_{\alpha}\right)=0, \quad 1 \leqq \alpha \leqq m$. But, $\quad 1=Q\left(E_{1}\right)+\ldots+Q\left(E_{m}\right)$. Suppose then that $Q\left(E_{\beta}\right) \neq 0$. Therefore $\delta_{\beta}=0$ and $E_{\beta}$ must be a finite set (since $C$ is non-trivial). But, then, $|\eta|=Q$ on $E_{\beta}$ and we have a contradiction.

We next study the ordinary linear extremal problem 


$$
\sup \left|\int_{E} f d \eta\right|=M(\eta)
$$

for $f \in A B(D),|f(z)| \leqq 1$. We claim that $M(\eta) \neq 0$. To prove this, we suppose first that $\eta\left(E_{1}\right)=\ldots=\eta\left(E_{m}\right)=0$, but $|\eta|\left(E_{\beta}\right) \neq 0$. Therefore,

$$
\int_{\partial D}\left|\omega_{\eta}+\varphi_{\infty}\right||d z|+\delta_{\alpha}|\eta|\left(E_{\alpha}\right) \leqq 0 .
$$

Hence, $\delta_{\beta}=0$ and $E_{\beta}$ is a finite set (since $C$ is non-trivial). By virtue of assumption (viii) in section $2, E$ is a Runge set for $A B(D)$ functions; see $[24$, p. 15]. We may thus find functions $f \in A B(D)$ which approximate 0 on $E-E_{\beta}$ and $|d \eta|(d \eta)^{-1}$ on the finite set $E_{\beta}$. Note here that $|\eta|\left(E_{\beta}\right)=$ $\sum_{x \in E_{\beta}}|\eta(x)|$. For such functions $f$, clearly $\int_{E} f d \eta \neq 0$. Hence $M(\eta) \neq 0$.

On the other hand, suppose that we have $\eta\left(E_{\beta}\right) \neq 0$ for some $\beta$. We can then find $f \in A B(D)$ which approximate 0 on $E-E_{\beta}$ and 1 on $E_{\beta}$. Again, $\int_{E} f d \eta \neq 0$, and $M(\eta) \neq 0$.

Now, choose any $f \in A B(D),|f(z)| \leqq 1$. Then,

$$
\begin{aligned}
& \operatorname{Re} \int_{\partial D} f\left(-\omega_{\eta}\right) d z+\operatorname{Re}\left\{a_{\alpha} \eta\left(E_{\alpha}\right)\right\}+\delta_{\alpha}|\eta|\left(E_{\alpha}\right) \\
& =\operatorname{Re} \int_{\partial D} f\left(-\omega_{\eta}-\varphi_{\infty}\right) d z+\operatorname{Re}\left\{a_{\alpha} \eta\left(E_{\alpha}\right)\right\}+\delta_{\alpha}|\eta|\left(E_{\alpha}\right) \\
& \leqq \int_{\partial D}\left|-\omega_{\eta}-\varphi_{\infty}\right||d z|+\operatorname{Re}\left\{a_{\alpha} \eta\left(E_{\alpha}\right)\right\}+\delta_{\alpha}|\eta|\left(E_{\alpha}\right) \\
& \leqq 0
\end{aligned}
$$

On the other hand, suppose that $f \in \mathscr{C}$. Then,

$$
\begin{aligned}
& \operatorname{Re} \int_{\partial D} f\left(-\omega_{\eta}\right) d z+\operatorname{Re}\left\{a_{\alpha} \eta\left(E_{\alpha}\right)\right\}+\delta_{\alpha}|\eta|\left(E_{\alpha}\right) \\
& =-\operatorname{Re} \int_{E} f d \eta+\operatorname{Re}\left\{a_{\alpha} \eta\left(E_{\alpha}\right)\right\}+\delta_{\alpha}|\eta|\left(E_{\alpha}\right)
\end{aligned}
$$

$\geqq 0$.

Thus, for every $f \in \mathscr{C}$,

$$
\operatorname{Re} \int_{\partial D} f\left(-\omega_{\eta}-\varphi_{\infty}\right) d z=\int_{\partial D} 1-\omega_{\eta}-\varphi_{\infty}|| d z \mid,
$$


so that $f\left(-\omega_{\eta}-\varphi_{\infty}\right) d z=\left|\omega_{\eta}+\varphi_{\infty}\right||d z|$ along $\partial D$. Since $M(\eta) \neq 0$, we must have $\omega_{\eta}+\varphi_{\infty} \neq 0$ along $\partial D$. Using the Lusin-Riesz-Priwalow theorem, we quickly deduce that $\ell$ is trivial. Contradiction.

It follows finally that $\left\|\mu_{n}\right\|$ must be bounded whenever

$$
\int_{\partial D}\left|k-\omega_{\mu_{n}}-\varphi_{n}\right||d z|+\operatorname{Re}\left\{a_{\alpha} \mu_{n}\left(E_{\alpha}\right)\right\}+\delta_{\alpha}\left|\mu_{n}\right|\left(E_{\alpha}\right) \rightarrow M_{0} .
$$

The boundedness of $\int_{\partial D}\left|\varphi_{n}(z)\right||d z|$ is now immediate.

Proof(Theorem 3). Let us first check that $M_{0}$ is actually a minimum when $C$ is non-trivial. To do so, choose any minimizing sequence $\left(\mu_{n}, \varphi_{n}\right)$ for problem $M_{0}$ and apply Theorem 2 . We may therefore assume wlog

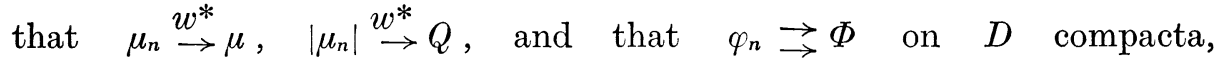
$\Phi \in H_{1}(D)$. We know too that $|\mu| \leqq Q$. By a simple extension of Lemma 2 , we see that

$$
\int_{\partial D}\left|k-\omega_{\mu}-\Phi\right||d z|+\operatorname{Re}\left\{a_{\alpha} \mu\left(E_{\alpha}\right)\right\}+\delta_{\alpha} Q\left(E_{\alpha}\right) \leqq M_{0}
$$

whence

$$
\int_{\partial D}\left|k-\omega_{\mu}-\Phi\right||d z|+\operatorname{Re}\left\{a_{\alpha} \mu\left(E_{\alpha}\right)\right\}+\delta_{\alpha}|\mu|\left(E_{\alpha}\right) \leqq M_{0} .
$$

It follows at once that $M_{0}$ is a minimum.

We must next show that $M=M_{0}$ whenever $C$ is non-empty. We shall first prove this in the case where $K$ is finite, $K \cap E=\phi$, and $€$ is non-trivial.

If $\lambda=0$, the result is obvious. Suppose therefore that $\lambda \neq 0$ and that $(\eta, \Phi)$ is extremal data for problem $M_{0}$. We maintain that $k \neq \omega_{\eta}+\Phi$ along $\partial D$. Otherwise, by the Lusin-Riesz-Priwalow theorem and assumption (viii), we see that $k(z) \equiv \omega_{\eta}+\Phi$ on $D-E$. Therefore the rational function $k(z)$ has no poles in $K$ and we deduce that $\lambda=0$. Contradiction.

Let us next define

$$
\sup \left|\int_{\partial D} f\left(k-\omega_{\eta}\right) d z\right|=\eta l
$$

for $f \in A B(D),|f(z)| \leqq 1$. We claim that $\eta \neq 0$. If not, then $\eta=0$ and Lemma 3 shows that $k-\omega_{\eta} \equiv q(z)$ near $\partial D$, with $q \in A B(D)$. Therefore $(\eta, q)$ is extremal data for problem $M_{0}$ and this contradicts the preceding paragraph. Hence, $n \neq 0$. 
Theorem 1 is now applicable to the ordinary linear extremal problem corresponding to kernel

$$
\tilde{k}=k-\omega_{\eta}
$$

and family $\{f \in A B(D):|f(z)| \leqq 1$ on $D\}$. Let $F$ be the normalized extremal function. Using remark 1 in section 4 , we see that

$$
\begin{aligned}
\Upsilon & =\inf _{\varphi \in H_{1}(D)} \int_{\partial D}|\tilde{k}-\varphi||d z|=\int_{\partial D}|\tilde{k}-\Phi||d z| \\
& =M_{0}-\operatorname{Re}\left\{a_{\alpha} \eta\left(E_{\alpha}\right)\right\}-\delta_{\alpha}|\eta|\left(E_{\alpha}\right) .
\end{aligned}
$$

We also know that $F(\tilde{k}-\Phi) d z=|\tilde{k}-\Phi||d z|$ along $\partial D$ and that $\Phi$ remains analytic across $\partial D$.

We shall now prove that $F \in \mathcal{C}$ by variational methods. Choose any point $z_{0} \in E_{1}$, let $\varepsilon_{0}$ denote the unit point mass at $z_{0}$, and let $\omega_{0}$ be the Cauchy transform of $\varepsilon_{0}$. Since $\left|\eta+t \varepsilon_{0}\right|\left(E_{1}\right) \leqq|\eta|\left(E_{1}\right)+|t|$, we see that

$$
\begin{gathered}
\int_{\partial D}\left|k-\omega_{\eta}-\varphi-t \omega_{0}\right||d z|+\operatorname{Re}\left\{a_{\alpha} \eta\left(E_{\alpha}\right)\right\}+\operatorname{Re}\left\{a_{1} t\right\} \\
+\delta_{\alpha}|\eta|\left(E_{\alpha}\right)+\delta_{1}|t| \geqq M_{0}
\end{gathered}
$$

for all $\varphi \in H_{1}(D)$ and $t \in \mathbf{C}$. Therefore

$$
\inf _{\varphi \in H_{1}(D)} \int_{\partial D}\left|\tilde{k}-t \omega_{0}-\varphi\right||d z| \geqq \eta-\delta_{1}|t|-\operatorname{Re}\left\{a_{1} t\right\} .
$$

Let

$$
\Upsilon_{t}=\sup \left|\int_{\partial D} f\left(\tilde{k}-t \omega_{0}\right) d z\right|
$$

for $f \in A B(D),|f(z)| \leqq 1$. Since $\chi \neq 0$, clearly $\bigcap_{t} \neq 0$ for $t \rightarrow 0$ and Theorem 1 applies. Let $F_{t}$ be the normalized extremal function. Therefore

$$
\eta_{t}=\int_{\partial D} F_{t}\left(\tilde{k}-t \omega_{0}\right) d z \geqq \eta-\operatorname{Re}\left\{a_{1} t\right\}-\delta_{1}|t| .
$$

Let $A_{t}=\int_{\partial D} F_{t} \tilde{k} d z$. Then $\left|A_{t}\right| \leqq \bigcap$. Moreover, since extremal function $F$ is unique, we readily check that $F_{t}(z) \rightrightarrows F(z)$ on $D$ compacta, so that $A_{t} \rightarrow \bigcap$. Thus, 


$$
\begin{gathered}
\left|A_{t}-t F_{t}\left(z_{0}\right)\right| \geqq \nearrow-\operatorname{Re}\left\{a_{1} t\right\}-\delta_{1}|t|, \\
\left.\left|A_{t}\right|^{2}-2 \operatorname{Re}\left\{t F_{t}\left(z_{0}\right) \bar{A}_{t}\right\}+O\left(|t|^{2}\right) \geqq \nearrow \chi^{2}-2^{c}\right\rceil\left[\operatorname{Re}\left(a_{1} t\right)+\delta_{1}|t|\right]+O\left(|t|^{2}\right), \\
-2 \operatorname{Re}\left\{t F_{t}\left(z_{0}\right) \bar{A}_{t}\right\} \geqq-2 \Upsilon\left[\operatorname{Re}\left(a_{1} t\right)+\delta_{1}|t|\right]+O\left(|t|^{2}\right) .
\end{gathered}
$$

Dividing by $|t|$ and letting $t=|t| e^{i \theta} \rightarrow 0$, we find that

$$
\operatorname{Re}\left\{\left[F\left(z_{0}\right)-a_{1}\right] e^{i \theta}\right\} \leqq \delta_{1}, \quad 0 \leqq \theta \leqq 2 \pi .
$$

Hence, $\left|F\left(z_{0}\right)-a_{1}\right| \leqq \delta_{1}$ for every $z_{0} \in E_{1}$. Similarly for the other $E_{k}$. It follows at once that $F \in \mathscr{E}$.

Let us next see what happens under a more general variation $\eta \rightarrow$ $\eta+t \sigma$, where $\sigma$ is a totally finite complex Borel measure on $R$, a compact subset of some $E_{\beta}$. We quickly deduce that

$$
\begin{gathered}
\int_{\partial D}\left|k-\omega_{\eta}-\varphi-t \omega_{\sigma}\right||d z|+\operatorname{Re}\left\{a_{\alpha} \eta\left(E_{\alpha}\right)\right\}+\delta_{\alpha}|\eta|\left(E_{\alpha}\right) \\
+\operatorname{Re}\left\{a_{\beta} t \sigma(R)\right\}+\delta_{\beta}|\eta+t \sigma|(R)-\delta_{\beta}|\eta|(R) \geqq M_{0}
\end{gathered}
$$

for all $\varphi \in H_{1}(D)$. Therefore,

$$
\begin{gathered}
\inf _{\varphi \in H_{1}(D)} \int_{\partial D}\left|\tilde{k}-t \omega_{\sigma}-\varphi\right||d z| \geqq \eta-\operatorname{Re}\left\{a_{\beta} t \sigma(R)\right\} \\
-\delta_{\beta}[|\eta+t \sigma|(R)-|\eta|(R)] .
\end{gathered}
$$

Reasoning as before, we find that for $t \rightarrow 0$

$$
\begin{gathered}
\sup _{|f| \leqq 1}\left|\int_{\partial D} f\left(\tilde{k}-t \omega_{\sigma}\right) d z\right| \geqq \eta-\operatorname{Re}\left\{a_{\beta} t \sigma(R)\right\} \\
-\delta_{\beta}[|\eta+t \sigma|(R)-|\eta|(R)]>0
\end{gathered}
$$

and we let $F_{t}$ be the extremal function. Once again, $F_{t} \rightarrow F,\left|A_{t}\right| \leqq \eta$, $A_{\imath} \rightarrow \eta$. We obtain

$$
\left|A_{t}-t \int_{R} F_{t} d \sigma\right| \geqq \nearrow-\operatorname{Re}\left\{a_{\beta} t \sigma(R)\right\}-\delta_{\beta}[|\eta+t \sigma|(R)-|\eta|(R)] .
$$

By squaring both sides, it follows that

$$
\begin{gathered}
-2 \operatorname{Re}\left\{\bar{A}_{t} t \int_{R} F_{t} d \sigma\right\} \geqq-2 \Upsilon\left[\operatorname{Re}\left\{a_{\beta} t \sigma(R)\right\}+\delta_{\beta}[|\eta+t \sigma|(R)-|\eta|(R)]\right] \\
+O\left(|t|^{2}\right) .
\end{gathered}
$$

This expression simplifies somewhat if we assume that 


$$
\sigma=\eta_{R},
$$

where $\eta_{R}$ denotes the restriction of $\eta$ to $R$. In that case,

$$
\begin{gathered}
\left.-2 \operatorname{Re}\left\{\bar{A}_{t} t \int_{R} F_{t} d \eta\right\} \geqq-2\right\urcorner 2\left[\operatorname{Re}\left\{a_{\beta} t \eta(R)\right\}+\delta_{\beta}|\eta|(R)\{|1+t|-1\}\right] \\
+O\left(|t|^{2}\right) .
\end{gathered}
$$

Dividing by $|t|$ and letting $t=|t| e^{i \theta} \rightarrow 0$, we find that

$$
-2 \operatorname{Re}\left\{\eta e^{i \theta} \int_{R} F d \eta\right\}+2 \nearrow \operatorname{Re}\left\{a_{\beta} \eta(R) e^{i \theta}+\delta_{\beta}|\eta|(R) e^{i \theta}\right\} \geqq 0,
$$

$0 \leqq \theta \leqq 2 \pi$.

Let us temporarily set $F=a_{\beta}+G_{\beta}$ on $E_{\beta}$. The preceding inequality yields

$$
\operatorname{Re}\left\{e^{i \ominus} \delta_{\beta}|\eta|(R)\right\} \geqq \operatorname{Re}\left\{e^{i \ominus} \int_{R} G_{\beta} d \eta\right\},
$$

from which we deduce that $G_{\beta} d \eta=\delta_{\beta} d|\eta|$ on $E_{\beta}$.

Finally, then, $F \in \mathcal{C}$ and

$$
\begin{aligned}
\operatorname{Re} \mathscr{L}(F) & =\operatorname{Re} \int_{\partial D} F\left(k-\omega_{\eta}-\Phi\right) d z+\operatorname{Re}\left\{a_{\alpha} \eta\left(E_{\alpha}\right)\right\}+\operatorname{Re} \int_{E_{\alpha}} G_{\alpha} d \eta \\
& =\int_{\partial D}\left|k-\omega_{\eta}-\Phi\right||d z|+\operatorname{Re}\left\{a_{\alpha} \eta\left(E_{\alpha}\right)\right\}+\delta_{\alpha}|\eta|\left(E_{\alpha}\right) \\
& =M_{\mathbf{0}} .
\end{aligned}
$$

Therefore $M_{0} \leqq M$, so that $M=M_{0}$.

We have thus proved that $M=M_{0}=$ minimum whenever $K$ is finite, $K \cap E=\phi$, and $\mathscr{C}$ is non-trivial.

We next look at the case where we know only that $e$ is non-trivial. The approximation argument used in the proof of Theorem 1 applies with trivial modifications. Thus, in obvious notation,

$$
\begin{aligned}
& \left|\mathscr{L}_{\varepsilon}(f)-\mathscr{L}(f)\right| \leqq \varepsilon\|f\| \int_{C}|k(t)||d t|, \quad f \in A B(D) ; \\
& M(\varepsilon)=M_{0}(\varepsilon)=\int_{\partial D}\left|k_{\varepsilon}-\omega_{\varepsilon}-\Phi_{\varepsilon}\right||d z|+\operatorname{Re}\left\{a_{\alpha} \eta_{\varepsilon}\left(E_{\alpha}\right)\right\}+\delta_{\alpha}\left|\eta_{\varepsilon}\right|\left(E_{\alpha}\right) ; \\
& \lim _{\varepsilon \rightarrow 0} M(\varepsilon)=M ; \\
& M_{0} \leqq M(\varepsilon)+\int_{\partial D}\left|k_{\varepsilon}-k\right||d z|,
\end{aligned}
$$


A. I. 586

and we conclude that $M_{0} \leqq M$, whence $M_{0}=M$.

Let us finally suppose that $e$ is trivial: $\mathscr{C}=\{F\}$. We define the family $\ell_{\varepsilon}=\left\{f \in A B(D):|f| \leqq 1+\varepsilon \quad\right.$ on $\quad D, \quad\left|f(z)-a_{k}\right| \leqq \delta_{k}+\varepsilon$ on $\left.E_{k}\right\}$. Since $F(z)+b \in \mathcal{C}_{\varepsilon}$ for $|b|<\varepsilon$, the family $\ell_{\varepsilon}$ is non-trivial. Using a trivial magnification, we conclude that $M\left[\mathcal{C}_{\varepsilon}\right]=M_{0}\left[\mathcal{C}_{\varepsilon}\right]$. In addition, a simple normal families argument shows that $\lim _{\varepsilon \rightarrow 0} M\left[\mathcal{C}_{\varepsilon}\right]=M$.

Now,

$$
\begin{gathered}
M_{0}\left[\mathscr{C}_{\varepsilon}\right]=\inf _{\mu, \varphi}\left[\int_{\partial D}(1+\varepsilon)\left|k-\omega_{\mu}-\varphi\right||d z|+\operatorname{Re}\left\{a_{\alpha} \mu\left(E_{\alpha}\right)\right\}+\right. \\
\left.+\left(\delta_{\alpha}+\varepsilon\right)|\mu|\left(E_{\alpha}\right)\right] .
\end{gathered}
$$

Therefore, $M_{0}\left[\mathcal{C}_{\varepsilon}\right] \geqq M_{0}$. Letting $\varepsilon \rightarrow 0$, we see that $M \geqq M_{0}$. Since $M \leqq M_{0}$ a priori, we see that $M=M_{0}$.

To complete the proof of Theorem 3, we must show that $M_{0}$ need not be a minimum when $\ell$ is trivial. This will be done in example $E$ in the next section.

\section{Further results and examples}

In this section, we shall examine a number of essentially straightforward consequences of Theorem 3 .

We shall use $(\eta, \Phi)$ to denote any solution of problem $M_{0}$ :

$$
M_{0}=\int_{\partial D}\left|k-\omega_{\eta}-\Phi\right||d z|+\operatorname{Re}\left\{a_{\alpha} \eta\left(E_{\alpha}\right)\right\}+\delta_{\alpha}|\eta|\left(E_{\alpha}\right) .
$$

Given such a pair $(\eta, \Phi)$, it is essential to distinguish the following two cases:

(I) $k-\omega_{\eta}-\Phi \neq 0$ near $\partial D$;

(II) $k-\omega_{\eta}-\Phi \equiv 0$ near $\partial D$.

The cases (I) and (II) are mutually exclusive.

Theorem 4. Suppose that $C$ is non-trivial and that case (I) holds. Then:

(i) there exists exactly one extremal function $F \in \mathscr{C}$ for problem $M$;

(ii) the two functions $\Phi$ and $F\left(k-\omega_{\eta}-\Phi\right)$ can be continued analytically across $\partial D$;

(iii) $F\left(k-\omega_{\eta}-\Phi\right) d z=\left|k-\omega_{\eta}-\Phi\right||d z|$ along $\partial D$; 
(iv) for each component $\Gamma$ of $\partial D$, there are exactly two possibilities:

(1) $k \neq \omega_{\eta}+\Phi$ near $\Gamma, F$ continues analytically across $\partial D$, and $|F|=1$ along $\Gamma$;

(2) $k \equiv \omega_{\eta}+\Phi$ near $\Gamma$, with nothing asserted about $F$.

Proof. We choose any extremal function $F \in \mathcal{C}$ and apply Theorem 3. Therefore

$$
\begin{aligned}
M=\operatorname{Re} \mathscr{L}(F) & =\operatorname{Re} \int_{\partial D} F k d z=\operatorname{Re} \int_{\partial D} F\left(k-\omega_{\eta}-\Phi\right) d z+\operatorname{Re} \int_{E} F d \eta \\
& =\operatorname{Re} \int_{\partial D} F\left(k-\omega_{\eta}-\Phi\right) d z+\operatorname{Re}\left\{a_{\alpha} \eta\left(E_{\alpha}\right)\right\}+\operatorname{Re}\left\{\int_{E_{\alpha}} G_{\alpha} d \eta\right\} \\
& \leqq \int_{\partial D}\left|k-\omega_{\eta}-\Phi\right||d z|+\operatorname{Re}\left\{a_{\alpha} \eta\left(E_{\alpha}\right)\right\}+\delta_{\alpha}|\eta|\left(E_{\alpha}\right) \\
& =M_{0}=M
\end{aligned}
$$

where $F=a_{\alpha}+G_{\alpha}$ on $E_{\alpha}$. It follows at once that $F\left(k-\omega_{\eta}-\Phi\right) d z=$ $\left|k-\omega_{\eta}-\Phi\right||d z|$ along $\partial D$ and that $G_{\alpha} d \eta=\delta_{\alpha} d|\eta|$ on $E_{\alpha}$.

Item (i) is now a consequence of the Lusin-Riesz-Priwalow theorem applied to $F_{1}\left(k-\omega_{\eta}-\Phi\right) d z=F_{2}\left(k-\omega_{\eta}-\Phi\right) d z$. And, of course, item (iii) is clear.

To prove the analyticity of $F\left(k-\omega_{\eta}-\Phi\right)$, one makes an auxiliary conformal mapping along $\partial D$, uses properties of the Smirnow class $E_{1}$, and applies the Schwarz reflection principle for harmonic functions of Hardy class $h_{1}$, just as in [15, p. 97].

The arguments for (iv) and the analyticity of $\Phi$ are exactly like those given in [15, pp. 97-99].

If $\mathscr{C}$ is non-empty, then the set $W=\{\mathscr{L}(f): f \in \mathcal{C}\}$ is closed and convex.

Theorem 5. Suppose that $C$ is non-trivial and that $W$ does not reduce to a point. Case $(\mathrm{I})$ is then valid under each of the following conditions:

(i) $k(z) \equiv R(z)$ near $\partial D$, where $R(z)$ is a rational function whose poles lie in $\mathbf{C}-E$;

(ii) $K \cap E=\phi$ and $\mathbf{C}-K$ is connected;

(iii) when all the $\delta_{k}=0$. 
Proof. Consider case (i) and suppose that case (II) holds. Using analytic continuation and assumption (viii) in section 2 , we see that $R(z) \equiv \omega_{\eta}+\Phi$ in $D-E$. It follows that $R(z)$ has no poles in $D$ and that $W=\{0\}$, which is a contradiction.

Suppose next that cases (ii) and (II) both hold. By analytic continuation, $k \equiv \omega_{\eta}+\Phi$ in $D-E \cup K$. But, then, $k(z)$ has a single-valued analytic continuation to all of $D$. Once again, $W=\{0\}$, which cannot be.

Suppose finally that all the $\delta_{k}=0$. Since $C$ is non-trivial, $E$ is a finite set. Suppose that case (II) holds. Then, for $f \in \mathscr{C}$,

$$
\mathscr{L}(f)=\int_{\partial D} f\left(k-\omega_{\eta}-\Phi\right) d z+a_{\alpha} \eta\left(E_{\alpha}\right)=a_{\alpha} \eta\left(E_{\alpha}\right)
$$

and we conclude that $W$ is a point. Contradiction.

Theorem 6. Suppose that $C$ is non-trivial and that problem $M$ has at least one non-constant extremal function. Then:

(i) extremal measure $\eta$ is concentrated on $\partial E$;

(ii) $\left|F(z)-a_{k}\right|=\delta_{k}$ a.e. [ $\left.\eta\right]$ on $E_{k}$ for every extremal function $F$.

Proof. Recall the first paragraph in the proof of Theorem 4. Therefore, $\left[F(z)-a_{k}\right] d \eta=\delta_{k} d|\eta|$ on $E_{k}$ for every extremal function $F$. But, one can write $d \eta=h d_{i} \eta \mid$ with a Borel measurable function $h,|h|=1$. Item (ii) follows by taking absolute values.

Suppose now that $F$ is a non-constant extremal function. Let $E_{k}^{0}$ denote the interior of $E_{k}$ and assume that $|\eta|\left(E_{k}^{0}\right) \neq 0$ for some $k$. By item (ii), $\left|F(z)-a_{k}\right|=\delta_{k}$ at least once in $E_{k}^{0}$. Since $\left|F(z)-a_{k}\right| \leqq \delta_{k}$ on $E_{k}$, the maximum modulus principle implies that $\left|F(z)-a_{k}\right| \equiv \delta_{k}$, whence $F(z) \equiv$ constant. From this contradiction, we conclude that $\eta$ is concentrated on $\partial E$.

Theorem 7. Suppose that problem $I I$ does not have a unique extremal function. Then:

(i) $C$ is non-trivial and case (II) holds;

(ii) extremal measure $\eta$ is concentrated at a finite number of points on $\partial E$;

(iii) $\mathscr{L}(f)$ assumes a very simple form:

$$
\mathscr{L}(f)=\sum_{a \in \partial E} \eta(a) f(a), \quad f \in A(D) .
$$


Proof. Item (i) follows from Theorem 4.

To prove (ii), we recall the first paragraph in the proof of Theorem 4 . Let $F(z)$ and $\tilde{F}(z)$ be two distinct extremal functions. Therefore $\left[F(z)-a_{k}\right] d \eta=\left[\tilde{F}(z)-a_{k}\right] d \eta=\delta_{k} d|\eta|$ on $E_{k}$. We write $d \eta=h d|\eta|$ with $|h|=1$, and let $T=\{z \in E: F(z)=\tilde{F}(z)\}$. Clearly, $(F-\tilde{F}) h d|\eta|=0$ on $E-T$, so that $|\eta|(E-T)=0$. Therefore, $\eta$ is concentrated on $T$, which is obviously finite. Suppose, however, that $\eta$ were not concentrated on $\partial E$. Then, by Theorem 6 , both $F$ and $\tilde{F}$ reduce to constants and $T$ must actually be empty.

Item (iii) follows at once from case (II) and (ii).

We shall now give five examples which illustrate our theorems.

Example $A$ (for case II). To begin with, let $D=\{1<|z|<2\}$, $K=\{|z|=3 / 2\}, \quad E=\{|z|=1+\varepsilon\}, \quad \varepsilon>0$ small, $\quad a=0, \quad 0<\delta(1+\varepsilon)$ $<1$, and

$$
\text { e }=\{f \in A B(D):|f(z)| \leqq 1 \text { on } D,|f(z)| \leqq \delta \text { on } E\} .
$$

Define

$$
\mathscr{L}(h)=\frac{1}{2 \pi i} \oint_{K} h(z) d z, h \in C(K) .
$$

An easy estimate shows that $|\mathscr{L}(f)| \leqq \delta(1+\varepsilon)$ for every $f \in \mathcal{C}$. Equality holds for $F(z)=\delta(1+\varepsilon) z^{-1} \in^{\complement} \ell$. Therefore $M=\delta(1+\varepsilon)$ and $F$ is an extremal function for problem $M$.

Observe, however, that $|F| \neq 1$ along $\partial D$. By Theorem 4, we conclude that case (II) holds. It is easy to check that $(d \eta, \Phi)=(d z / 2 \pi i, 0)$ gives extremal data for problem $M_{0}$. Finally, Theorem 7 shows that extremal function $F$ is unique.

Example $B$ (for case I). Let $D=\{1<|z|<3\}, \quad E=\{2\}, \quad K=$ $\{|z|=3 / 2\}$,

$$
\mathscr{C}=\{f \in A B(D):|f(z)| \leqq 1 \text { on } D, f(2)=1 / 2\}
$$

and

$$
\mathscr{L}(h)=\frac{1}{2 \pi i} \oint_{K} h(z) d z
$$

By a trivial deformation of path $K$, clearly $\operatorname{Re} \mathscr{L}(f) \leqq|\mathscr{L}(f)| \leqq 1$ for $f \in{ }^{C}$. Equality holds for $F(z)=z^{-1} \in \mathscr{C}$. Therefore $M=1$ and $F$ is an extremal function for problem $M$.

By means of Theorem 5, case (I) must hold. Using Theorem 4, we immediately conclude that $F$ is unique and that $k \equiv \omega_{\eta}+\Phi$ along $|z|=3$. 
Moreover, we easily check that $(\eta, \Phi)=(0,0)$ gives extremal data for dual problem $M_{0}$.

Example $C$ (for non-uniqueness). Let $\mathrm{D}=\{|z|<1\}, K=\{|z|=1-\varepsilon\}$, $\varepsilon>0$ small, $E=\{1 / 2\}$, and

$$
\mathscr{L}(h)=\frac{1}{2 \pi i} \oint_{K} \frac{h(z)}{z-1 / 2} d z .
$$

Choose any $0 \leqq a<1 / 4$ and let $\delta=1 / 4-a$. We easily check that the functions $F_{1}(z)=z / 2$ and $F_{2}(z)=z^{2}$ are both in ce and are extremal functions for problem $M(M=1 / 4)$.

It is thus apparent that Theorem 7 applies. For extremal data $(\eta, \Phi)$ we can take $\left(\varepsilon_{1 / 2}, 0\right)$, where $\varepsilon_{1 / 2}$ denotes the unit mass at $\{1 / 2\}$.

Example $D$ (for Theorem 6). Let $D=\{|z|<1\}, E=\{|z| \leqq 1 / 3\}$, $K=\{|z|=1 / 2\}$,

$$
\mathscr{C}=\{f \in A B(D):|f(z)| \leqq 1 \text { on } D,|f(z)| \leqq 1 / 3 \text { on } E\},
$$

and

$$
\mathscr{L}(h)=\frac{1}{2 \pi i} \oint_{K} \frac{h(z)}{z} d z .
$$

Clearly $F(z)=1 / 3$ is in $C$ and is extremal for problem $M(M=1 / 3)$.

However, we can take $(\eta, \Phi)=\left(\varepsilon_{0}, 0\right)$, where $\varepsilon_{0}$ is the unit mass at $\{0\}$. Thus, the existence of non-constant extremal functions is essential in Theorem 6.

Example $E$ (for $M_{0} \neq$ minimum). Let $D=\{|z|<1\}, E=\{|z| \leqq 1 / 3\}$, $K=\left\{z_{0}\right\}, \quad \frac{1}{2}<\left|z_{0}\right|<\frac{3}{4}, \quad a=\delta=0, \quad$ and $\quad \mathscr{L}(h)=h\left(z_{0}\right)$. Clearly $\mathscr{C}=\{0\}$. Using Theorem 3 , we know that $M=M_{0}=0$. However, $M_{0}$ is not a minimum. If it were, then

$$
0=\int_{\partial D}\left|k-\omega_{\eta}-\Phi\right||d z|,
$$

and we could conclude that $k=\omega_{\eta}+\Phi$ near $\partial D$. Therefore,

$$
\frac{1}{2 \pi i} \frac{1}{z-z_{0}} \equiv \omega_{\eta}+\Phi, \frac{1}{3}<|z|<1,
$$

which is impossible.

\section{Further discussion of Carleman-Milloux problems}

In this section, we shall give some indication of how Carleman-Milloux type problems can be handled when $E$ is allowed to intersect $\partial D$. This 
is the case, for example, in the classical Carleman-Milloux problem. We shall be content to study the following situation. We assume that $E$ is a Jordan arc contained entirely within $D$, except for the terminal point, which lies on $\partial D$. Further, assume that $K \cap E=\phi$ and that C $-K$ is connected. Let $0<\delta<1$ and define

$$
\mathcal{e}=\{f \in A B(D):|f(z)| \leqq 1 \text { on } D,|f(z)| \leqq \delta \text { on } E\} .
$$

To avoid the trivial case, assume finally that

$$
M=\sup \operatorname{Re} \mathscr{L}(f)=\sup |\mathscr{L}(f)| \neq 0, f \in \mathscr{\ell} .
$$

Let $E_{n}$ be an increasing sequence of compact subarcs of $E$ such that $\cup E_{n}=E \cap D$. We can then legitimately consider the extremal problem for $\mathscr{L}$ over $e_{n}$, where

$$
\bigodot_{n}=\left\{f \in A B(D):|f(z)| \leqq 1 \text { on } D,|f(z)| \leqq \delta \text { on } E_{n}\right\} .
$$

Using Theorems 4 and 5 , we determine the usual extremal data $M_{n}, F_{n}, \omega_{n}, \Phi_{n}, \eta_{n}$ so that

$$
M_{n}=\mathscr{L}\left(F_{n}\right)=\int_{\partial D}\left|k-\omega_{n}-\Phi_{n}\right||d z|+\delta\left\|\eta_{n}\right\|,
$$

where $\omega_{n}=\omega_{\eta_{n}}$ and $\| \eta_{n}|=| \eta_{n}\left(E_{n}\right)$. It is convenient to write

$$
S_{n}=\omega_{n}+\Phi_{n} \text {. }
$$

We know that both $F_{n}$ and $S_{n}$ continue analytically across $\partial D$, while $\left|F_{n}\right|=1$ and $F_{n}\left(k-S_{n}\right) d z=\left|k-S_{n}\right||d z|$ along $\partial D$. It is simple to check that $M_{n} \rightarrow M$ monotonically as $n \rightarrow \infty$.

Suppose now that $D_{1}$ is a smoothly bounded Jordan domain such that $K \subseteq D_{1} \subseteq D_{1} \cup \partial D_{1} \subseteq D-E$. It is convenient to define

$$
\begin{gathered}
I_{n}[f](z)=\frac{1}{2 \pi i} \int_{\partial D} \frac{f\left(k-S_{n}\right)}{\xi-z} d \xi-\frac{1}{2 \pi i} \int_{\partial D_{1}} \frac{f\left(k-S_{n}\right)}{\xi-z} d \xi \\
\quad+\frac{1}{2 \pi i} \int_{E_{n}} \frac{f(t)}{t-z} d \eta_{n}(t)
\end{gathered}
$$

for $f \in A B(D)$ and $z \in Z, \quad Z=\mathbf{C}-E-D_{1}-\partial D_{1}-\partial D$.

A simple computation shows that:

$$
\begin{gathered}
I_{n}[f](z)=\frac{1}{2 \pi i} \int_{\partial D} \frac{f\left(k-S_{n}\right)}{\xi-z} d \xi-\frac{1}{2 \pi i} \int_{K} \frac{f(t)}{t-z} d \lambda(t)+ \\
\quad+\frac{1}{2 \pi i} \int_{E_{n}} \frac{f(t)}{t-z} d \eta_{n}(t) ;
\end{gathered}
$$




$$
I_{n}[f](z)=\left\{\begin{array}{cl}
f(z)\left[k(z)-S_{n}(z)\right], & z \in Z \cap D \\
0 & , z \in Z-D .
\end{array}\right.
$$

Formula (b) shows that we can represent $f(z)\left[k(z)-S_{n}(z)\right]$ as a sort of Poisson integral, at least near $\partial D-E$; namely,

$$
f(z)\left[k(z)-S_{n}(z)\right]=I_{n}[f](z)-I_{n}[f]\left(z^{*}\right),
$$

where $z^{*}$ is simply the reflection of $z$ in $\partial D$.

Using the formulas for $I_{n}[1](z)$ and the fact that

$$
\int_{\partial D}\left|k-S_{n}\right||d z| \leqq M_{1}
$$

we quickly deduce that the functions $S_{n}(z)$ are uniformly bounded on $D-E \cup D_{1} \cup \partial D_{1}$ compacta. We may therefore assume wlog that

$$
\begin{aligned}
& F_{n}(z) \rightarrow F_{\infty}(z) \text { on } D \text { compacta; } \\
& S_{n}(z) \rightarrow S_{\infty}(z) \text { on } D-E \text { compacta. }
\end{aligned}
$$

Moreover, since $\left\|\eta_{n}\right\| \leqq M_{1} \delta^{-1}$, we may also assume that

$$
\eta_{n} \stackrel{w^{*}}{\rightarrow} \eta \text { on } E \text {. }
$$

Note that $\eta$ may well be concentrated at $E \cap \partial D$.

We must next study $F_{\infty}$ and $S_{\infty}$ in the neighborhood of an arbitrary point $P \in \partial D-E$. To do so, we choose neighborhoods $A_{k} \subseteq \partial D$ of $P$ so that

$$
A>A_{1}>A_{2}>A_{3}>P
$$

in an obvious way. Since the problem is now local in nature, we may (as is easily checked) assume wlog that $A \subseteq \mathbf{R}$ with $D$ situated above $A$.

By use of representation (c) above and writing $z=u+i v$, we readily obtain:

$$
F_{n}(z)\left[k(z)-S_{n}(z)\right]=\frac{v}{\tau} \int_{A} \frac{F_{n}\left(k-S_{n}\right)}{(x-u)^{2}+v^{2}} d x+O(v)
$$

for $z \in D$ near $A_{1}$, uniformly in $n$.

By the Schwarz reflection principle, the functions $F_{n}\left(k-S_{n}\right)$ are all analytic in a fixed rectangular neighborhood $R_{1}$ of $A_{1}$. By means of the above integral representation and the bound on $\int_{A}\left|k-S_{n}\right||d z|$, we find that

$$
\int_{R_{1} \cap D} \int_{D}\left|F_{n}(z)\right|\left|k(z)-S_{n}(z)\right| d u d v \leqq C_{1}
$$


independently of $n$. We let $R_{1} \cap \partial D=\left(x_{1}, x_{2}\right) \subseteq \mathbf{R}$ and introduce polar coordinates $z=x_{j}+r e^{i \theta}, j=1,2$. Then wlog

$$
\int_{I} \int_{0}^{R}\left|F_{n}\right|\left|k-S_{n}\right| r d r d \theta \leqq C_{1},
$$

where $I=\left[\frac{1}{4} \pi, \frac{3}{4} \pi\right]$ and $R$ depends only on $R_{1}$. It follows that

$$
\min _{\theta \in I} \int_{0}^{R}\left|F_{n}\right|\left|k-S_{n}\right| r d r \leqq C_{2},
$$

independently of $n$. By means of the Cauchy formula and the Schwarz reflection principle, we easily see that the functions

$$
F_{n}\left(k-S_{n}\right)\left(z-x_{1}\right)\left(z-x_{2}\right)
$$

are uniformly bounded in a fixed rectangular neighborhood $R_{2}$ of $A_{2}$.

A similar argument using $k-S_{n}$ in place of $F_{n}\left(k-S_{n}\right)$ leads to the estimate

$$
\min _{\theta \in I} \int_{0}^{R}\left|k-S_{n}\right| r d r \leqq C_{3} .
$$

Since $F_{n}\left(k-S_{n}\right) d x \geqq 0$ and $\left|F_{n}\right|=1$ along $A$, two applications of the Schwarz reflection principle will show that $S_{n} \in A\left(R_{1}\right)$ and that

$$
\left|k(\bar{z})-S_{n}(\bar{z})\right|=\left|F_{n}(z)\right|^{2}\left|k(z)-S_{n}(z)\right| \leqq\left|F_{n}(z)\right|\left|k(z)-S_{n}(z)\right|
$$

for $z \in R_{1} \cap D$. The $C_{2}$ estimate now yields one for the integrals $\int\left|k-S_{n}\right| r d r$ below the segment $A \subseteq \mathbf{R}$. It follows at once that the functions $\left(k-S_{n}\right)\left(z-x_{1}\right)\left(z-x_{2}\right)$ are also uniformly bounded on $R_{2}$.

A standard normal families argument now shows that both $S_{\infty}$ and $F_{\infty}\left(k-S_{\infty}\right)$ have analytic continuations to all of $R_{2}$. Moreover, the following uniform limits hold on $R_{2}$ :

$$
\begin{aligned}
S_{\infty} & =\lim _{n \rightarrow \infty} S_{n} ; \\
F_{\infty}\left(k-S_{\infty}\right) & =\lim _{n \rightarrow \infty} F_{n}\left(k-S_{n}\right) .
\end{aligned}
$$

Recall here that $S_{n}$ and $F_{n}\left(k-S_{n}\right)$ are known to be in $A\left(R_{1}\right)$.

We shall now prove the following important

Fact: $\left|F_{\infty}(x)\right|=1$ a.e. on $A_{3}$. 
Proof. Suppose not. There must then exist a compact set $Q \subseteq A_{3}$, $m(Q) \neq 0$, on which $\left|F_{\infty}(x)\right| \leqq 1-\tau, \tau>0$. However,

$$
\begin{aligned}
M & =\mathscr{L}\left(F_{\infty}\right)=\operatorname{Re} \int_{\partial D} F_{\infty}\left(k-S_{n}\right) d z+\operatorname{Re} \int_{E_{n}} F_{\infty} d \eta_{n} \\
& \leqq \int_{\partial D}\left|F_{\infty}\right|\left|k-S_{n}\right||d z|+\delta\left\|\eta_{n}\right\| \\
& \leqq \int_{\partial D}\left|k-S_{n}\right||d z|+\delta\left\|\eta_{n}\right\|=M_{n} \rightarrow M .
\end{aligned}
$$

Therefore, $\lim _{n \rightarrow \infty} \int_{Q}\left[1-\left|F_{\infty}\right|\right]\left|k-S_{n}\right||d z|=0$, whence

$$
\lim _{n \rightarrow \infty} \int_{Q}\left|k-S_{n}\right||d z|=0 .
$$

But, $S_{n} \rightarrow S_{\infty}$ on $R_{2}$. Therefore, $\int_{Q}\left|k-S_{\infty}\right||d z|=0$ and we see that $k \equiv S_{\infty}$, which leads to the contradiction $M=0$.

We can now apply the classical factorization theorem of Szegö-Smirnow [22, p. 78] to the functions $0 \neq F_{\infty}\left(k-S_{\infty}\right) \in A\left(R_{2}\right), \quad F_{\infty} \in A B(D)$, and $S_{\infty} \in A\left(R_{2}\right)$. Using the fact, we conclude that $F_{\infty}$ continues analytically across $A_{3}$ and that $\left|F_{\infty}\right|=1$ everywhere along $A_{3}$. See also [15, p. 98].

Suppose now that $F$ is any extremal function: $\mathscr{L}(F)=M$. By repeating the proof of the fact, we see that $|F(x)|=1$ a.e. on $A_{3}$. But, $\frac{1}{2}\left(F+F_{\infty}\right)$ is also extremal. Therefore

$$
\left|F+F_{\infty}\right|=|F|+\left|F_{\infty}\right|=2 \text { a.e. on } A_{3} .
$$

This implies that $F=F_{\infty}$ a.e. on $A_{3}$. By the Lusin-Riesz-Priwalow theorem, we conclude that $F \equiv F_{\infty}$.

We can summarize the preceding results as follows:

Theorem 8. The Carleman-MIilloux extremal problem posed above has a unique solution $F$. There also exists an analytic function $S$ on $D-E$ such that:

(i) both $F$ and $S$ continue analytically across $\partial D-E$;

(ii) $|F|=1$ along $D-E$;

(iii) $F(k-S) d z=|k-S||d z|$ along $\partial D-E$;

(iv) $\int_{\partial D-E}|k-S||d z| \leqq M$.

A similar theorem clearly holds whenever $E$ is a compact subset of C such that $D-E$ is connected. See also $[9, \mathrm{pp} .69-71]$. 


\section{Further discussion of Pick-Nevanlinna problems}

We shall be concerned here with the following

Problem. Suppose that:

(a) assumptions (i), (iii), (iv), (v), and (vi) of section 2 apply;

(b) $E$ is a sequence of distinct points $\xi_{k} \in D$ tending to $\xi_{\infty} \in \partial D$;

(c) $K \cap E=\phi$ and $\mathbf{C}-K$ is connected;

(d) $\mathscr{C}=\left\{f \in A B(D):|f(z)| \leqq 1\right.$ on $\left.D, f\left(\xi_{k}\right)=a_{k}\right\}$;

(e) $\mathcal{C}$ is non-trivial;

(f) the set $W=\{\mathscr{L}(f): f \in \mathscr{C}\}$ does not reduce to a point;

(g) $M=\sup \operatorname{Re} \mathscr{L}(f)$ over $f \in \mathcal{C}$.

We want to describe the extremal functions $F \in \mathcal{C}$ which satisfy

$$
\operatorname{Re} \mathscr{L}(F)=M \text {. }
$$

The method we use is quite similar to that of section 7 , only somewhat more difficult. In this context, then, we introduce $E_{n}=\left\{\xi_{1}, \ldots, \xi_{n}\right\}$,

$$
e_{n}=\left\{f \in A B(D):|f(z)| \leqq 1 \text { on } D, f\left(\xi_{k}\right)=a_{k} \text { for } 1 \leqq k \leqq n\right\},
$$

and the extremal data $M_{n}, F_{n}, \omega_{n}, \Phi_{n}, \eta_{n}$ so that

$$
M_{n}=\operatorname{Re} \mathscr{L}\left(F_{n}\right)=\int_{\partial D}\left|k-\omega_{n}-\Phi_{n}\right||d z|+\sum_{k=1}^{n} \operatorname{Re}\left\{a_{k} \eta_{n}\left(\xi_{k}\right)\right\} .
$$

We also introduce $S_{n}=\omega_{n}+\Phi_{n}$ and the Jordan domain $D_{1}$. As before, $M_{n} \rightarrow M$ monotonically as $n \rightarrow \infty$.

We now try to extend the argument used in section 7 . It is important to get bounds on $\int_{\partial D}\left|k-S_{n}\right||d z|$ and $S_{n}(z)$. Observe, however, that it is conceivable that

$$
\begin{aligned}
& \lim _{n \rightarrow \infty} \int_{\partial D}\left|k-S_{n}\right||d z|=+\infty ; \\
& \lim _{n \rightarrow \infty} \sum_{k=1}^{n} \operatorname{Re}\left\{a_{k} \eta_{n}\left(\xi_{k}\right)\right\}=-\infty .
\end{aligned}
$$

To get around this obstacle, we observe that

$$
M_{n}-\operatorname{Re} \mathscr{L}(f) \geqq \int_{\partial D}[1-|f|]\left|k-S_{n}\right||d z|
$$

for every $f \in \mathcal{C}$. Let $f_{1}$ and $f_{2}$ be two distinct functions in $\mathcal{C}$. We can then write $f_{1}=f+q, f_{2}=f-q$ with $f \in \mathscr{C}$ and $q \neq 0$. Moreover, 
since $|f \pm q| \leqq 1$, we see that $|f|^{2}+|q|^{2} \leqq 1$. If we now let $Q(z)=$ $\frac{1}{2} q(z)^{2}$, we find that: (i) $Q(z) \neq 0$; (ii) $\|Q\| \leqq \frac{1}{2}$; (iii) $Q\left(\xi_{k}\right)=0$; (iv) $|f(z)|+|Q(z)| \leqq 1$. Hence, for some constant $B$ and all $n$,

$$
\int_{\partial D}|Q|\left|k-S_{n}\right||d z| \leqq B
$$

By studying $I_{n}[Q](z)$, we quickly deduce that $Q S_{n}$ is uniformly bounded on $D-E-D_{1}-\partial D_{1}$ compacta. Using $Q \neq 0$ and the maximum modulus principle, we see that $S_{n}(z)$ is uniformly bounded on $D-E$ compacta. Therefore, wlog,

$$
\begin{aligned}
F_{n}(z) & \rightarrow F_{\infty}(z) \text { on } D \text { compacta; } \\
S_{n}(z) & \rightarrow S_{\infty}(z) \text { on } D-E \text { compacta. }
\end{aligned}
$$

We will not worry about the measures $\eta_{n}$.

We now study the local behavior of $F_{\infty}$ and $S_{\infty}$ by means of the neighborhoods

$$
A>A_{1}>A_{2}>A_{3}>P \in \partial D-E .
$$

Once again, we assume wlog that $A \subseteq \mathbf{R}$. Using $I_{n}\left[F_{n} Q\right](z)$, we find that

$$
Q(z) F_{n}(z)\left[k(z)-S_{n}(z)\right]=\frac{v}{\tau} \int_{A} \frac{Q F_{n}\left(k-S_{n}\right)}{(x-u)^{2}+v^{2}} d x+O(v)
$$

for $z \in D$ near $A_{1}$, uniformly in $n$.

By the Schwarz reflection principle, the functions $F_{n}\left(k-S_{n}\right)$ all continue analytically to some fixed rectangular neighborhood $R_{1}$ of $A_{1}$. By shrinking $R_{1}$ somewhat, we may assume that: (i) $R_{1} \cap \partial D=$ $\left(x_{1}, x_{2}\right) \subseteq \mathbf{R}$; (ii) the non-tangential limits $Q\left(x_{1}\right)$ and $Q\left(x_{2}\right)$ exist and are both non-zero. It follows that $|Q(z)|$ is bounded away from 0 in the sectors $\frac{1}{4} \pi<\arg \left(z-x_{i}\right)<\frac{3}{4} \pi$ as $z \rightarrow x_{i}$.

The integral representation implies that

$$
\int_{R_{1} \cap D} \int_{D}\left\{Q: F_{n}:\left[k-S_{n} \mid d u d v \leqq B_{1}\right.\right.
$$

independently of $n$. By introducing polar coordinates $z=x_{i}+r e^{i \theta}$, we see that wlog

$$
\int_{I} \int_{0}^{R}|Q|\left|F_{n}\right|\left|k-S_{n}\right| r d r d \theta \leqq B_{1},
$$

where $I=\left[\frac{1}{4} \pi, \frac{3}{4} \pi\right]$ and $R$ is independent of $n$. It follows that 


$$
\min _{\theta \in I} \int_{0}^{R}|Q|\left|F_{n}\right|\left|k-S_{n}\right| r d r \leqq B_{2}
$$

independently of $n$. By shrinking $R$ somewhat, we may clearly assume that

$$
\min _{\theta \in I} \int_{0}^{R}\left|F_{n}\right|\left|k-S_{n}\right| r d r \leqq B_{3} .
$$

By the Cauchy formula and the Schwarz reflection principle, we find that the functions $F_{n}\left(k-S_{n}\right)\left(z-x_{1}\right)\left(z-x_{2}\right)$ are uniformly bounded in some fixed rectangular neighborhood $R_{2}$ of $A_{2}$.

Observe that a similar argument holds when $Q F_{n}\left(k-S_{n}\right)$ is replaced by just $Q\left(k-S_{n}\right)$. We thus find that

$$
\min _{\theta \in I} \int_{0}^{R}\left|k-S_{n}\right| r d r \leqq B_{4} .
$$

As in section 7, the Schwarz reflection principle implies that $S_{n} \in A\left(R_{1}\right)$ and that $\left|k(\bar{z})-S_{n}(\bar{z})\right|=\left|F_{n}(z)\right|^{2}\left|k(z)-S_{n}(z)\right| \leqq\left|F_{n}(z)\right|\left|k(z)-S_{n}(z)\right|$ for $z \in R_{1} \cap D$. The $B_{3}$ estimate now leads to one for the integrals $\int\left|k-S_{n}\right| r d r$ below the segment $A \subseteq \mathbf{R}$. The functions

$$
\left(k-S_{n}\right)\left(z-x_{1}\right)\left(z-x_{2}\right)
$$

are therefore uniformly bounded on $R_{2}$.

The usual normal families argument shows that $S_{n}(z) \rightrightarrows S_{\infty}(z)$ and $F_{n}\left(k-S_{n}\right) \rightarrow F_{\infty}\left(k-S_{\infty}\right)$ on $R_{2}$.

The remainder of the argument follows section 7 almost verbatim. To prove that $\left|F_{\infty}(x)\right|=1$ a.e. on $A_{3}$, one uses

$$
M_{n}-M \geqq \int_{\partial D}\left[1-\left|F_{\infty}\right|\right]\left|k-S_{n}\right||d z| .
$$

It is easily verified that $F_{\infty}\left(k-S_{\infty}\right)=0$ leads to the contradiction $W=\{0\}$.

Our conclusion is as follows:

Theorem 9. The Pick-Nevanlinna problem posed above has a unique solution $F$. There also exists a meromorphic function $S$ on $D$, having at most simple poles on $E$, such that:

(i) both $F$ and $S$ continue analytically across $\partial D-E$;

(ii) $|\boldsymbol{F}|=1$ along $\partial D-E$;

(iii) $F(k-S) d z=|k-S||d z|$ along $\partial D-E$. 
The remark about the poles of $S(z)$ follows from the uniform boundedness of $Q S_{n}$ proved above.

It is also clear that a similar theorem holds for much more general sets $E$.

In certain cases, it is possible to prove that the extremal function is unique with very little work. Suppose, for example, that $\mathscr{L}(f)=f\left(z_{0}\right)$, where $z_{0} \neq$ all $\xi_{k}$. Let $F_{1}$ and $F_{2}$ be two distinct extremal functions: $\operatorname{Re} \mathscr{L}\left(F_{1}\right)=\operatorname{Re} \mathscr{L}\left(F_{2}\right)=M$. We may therefore write $F_{1}=F+Q$, $F_{2}=F-Q$, where $F$ is also extremal and $Q \equiv 0$. Since $|F \pm Q| \leqq 1$, we see that $|F|^{2}+|Q|^{2} \leqq 1$. Forming $g(z)=Q(z)^{2} / 2$, we find that: (i) $g \equiv 0$; (ii) $\|g\| \leqq 1 / 2$; (iii) $g\left(\xi_{k}\right)=0$; and (iv) $|F(z)|+|g(z)| \leqq 1$. Write $g(z)=\left(z-z_{0}\right)^{m} g_{1}(z)$ with $g_{1}\left(z_{0}\right) \neq 0$ and define

$$
h(z)=F(z)+R e^{i \theta} g_{1}(z) .
$$

For $R \rightarrow 0$, one readily proves that $h \in \mathcal{C}$ (using the maximum modulus principle). Therefore $\operatorname{Re}\left[e^{i \theta} g_{1}\left(z_{0}\right)\right] \leqq 0$ and we deduce the contradiction $g_{1}\left(z_{0}\right)=0$. This method of proof is due to S. Fisher [4].

\section{Generalizations and open problems}

The arguments we have used in previous sections can of course be generalized considerably. It may be of interest to mention a few of these generalizations explicitly.

We begin by recalling the statement of our problem in section 2. From this, we see that there are at least six possible directions for generalization:

(a) the restriction $|f(z)| \leqq 1$;

(b) the restriction $\left|f(z)-a_{k}\right| \leqq \delta_{k}$;

(c) the set $K$;

(d) the linear functional $\mathscr{L}$;

(e) the set $E$;

(f) the domain $D$.

Regarding direction (a), it is entirely possible to replace the (boundary) condition $|f(z)| \leqq 1$ by a more general one, such as $\ln |f| \leqq \chi$, where $\chi$ is some harmonic function. See [15].

With regard to (b), it is possible to use conditions of the form

$$
\left|\ell_{k}(f)-a_{k}\right| \leqq \delta_{k},
$$

where the $\ell_{k}$ are linear functionals (e.g. derivatives). Furthermore, one can allow $a_{k}$ and $\delta_{k}$ to be functions and can consider other norms (such as $L_{p}$ ). The articles by Havinson [9] and Gamelin [6] are of particular interest here. 
The restriction that $K$ be a compact subset of $D$ can be relaxed somewhat. The important thing is that we ultimately obtain a representation $\mathscr{L}(f)=\int_{\partial D} f(z) k(z) d z$ with a reasonable kernel $k(z)$.

One might also wish to examine non-linear functionals $\mathscr{L}$. It would be interesting to see how dual extremal problems fit into the picture. However, not too much appears to be known about such problems. See also [5] and [11].

We now turn to direction (e). We have already given some indication of what happens when $E \cap \partial D$ is nonvoid, at least for Carleman-Milloux and Pick-Nevanlinna problems. The main point is that the dual extremal problem of section 5 becomes singular and one is forced to reason by approximation. It may also be of interest to study the dual function $S(z)$ more deeply.

Direction (f) is concerned with the domain $D$ itself. One would like to know what happens to our extremal problems on arbitrary plane domains $D$. The situation for ordinary linear extremal problems was investigated in [15]. Using similar methods, together with those of sections 7 and 8 , we have developed a reasonably complete theory for certain cases, including the Carleman-Milloux and Pick-Nevanlinna. In doing so, we use exhaustions $D_{n} \uparrow D$ and $E_{n} \uparrow E$, where the $D_{n}$ are smoothly bounded and $E_{n}$ is a compact subset of $D_{n}$. (These $E_{n}$ have nothing to do with the old $E_{k}$.)

In a slightly different direction, one may wish to replace the domain $D$ by an open Riemann surface $W$. If $W$ is a smoothly bounded subregion of a compact surface $W_{0}$, there seems to be a reasonable theory. See also [1], [12], and [23]. However, the question of linear extremal problems on arbitrary open surfaces $W$, especially those of infinite genus, is rather poorly understood at present. Compare [15, pp. 117-121].

One obvious problem which has not been mentioned at all in the preceding sections concerns the uniqueness of the dual extremal data $(\eta, \Phi)$. Information about this problem can be found in [10].

We mention one last area, which seems to be particularly interesting. This area concerns explicit formulas. As is well-known [7], the solution of the Schwarz lemma problem can be expressed in terms of the Szegö kernel function (see also [2, pp. 86-89]). One would like to determine whether a similar interpretation exists for the solutions of Pick-Nevanlinna problems. The proper context for such questions appears to be in terms of the compact surface $R$ formed by doubling $D$. This stems from the fact that the differential $F\left(k-\omega_{\eta}-\Phi\right) d z$ and the function $F$ actually »live» on $R$, by virtue of the Schwarz reflection principle. Such methods lead one to Jacobi inversion problems for integrals of the third kind on $R$, as observed by Garabedian [7, pp. 30-31]. See also [16]. 
A. I. $\quad 586$

The situation for Carleman-Milloux problems is admittedly less hopeful. However, in the classical case, Heins [13] proved that the solution can be expressed as the quotient of two theta functions. It would be extreme $\mathrm{y}$ interesting to fit this representation into some sort of larger framework.1

\author{
Harvard University \\ Department of Mathematics \\ Cambridge, Mass. 02138, USA
}




\section{References}

[1] Ahlfors, L. V.: Open Riemann surfaces and extremal problems on compact subregions, Comment. Math. Helv. 24 (1950) pp. 100-134.

[2] Bergman, S.: The Kernel Function and Conformal Mapping, Amer. Math. Soc. Math. Surveys 5, 1950.

[3] Carleson, L.: Selected Problems on Exceptional Sets, Van Nostrand, Princeton, 1967.

[4] Fisher, S.: On Schwarz's lemma and inner functions, Trans. Amer. Math. Soc. 138 (1969) pp. 229-240.

[5] Fisher, S.: Non linear extremal problems in $H^{\infty}$, Indiana Univ. Math. J. 22 (1973) pp. 1183-1190.

[6] Gamelin, T.: Extremal problems in arbitrary domains, Michigan Math. J. 20 (1973) pp. 3-11.

[7] Garabedian, P. R.: Schwarz's lemma and the Szegö kernel function, Trans. Amer. Math. Soc. 67 (1949) pp. 1-35.

[8] Golusin, G. M.: Geometrische Funktionentheorie, VEB Deutscher Verlag der Wissenschaften, Berlin, 1957.

[9] Havinson, S. JA.: Extremal problems for bounded analytic functions with interior side conditions, Russian Math. Surveys 18 (1963) no. 2, pp. 23 $-96$.

[10] -》- Extremal problems for certain classes of analytic functions in finitely connected regions, Amer. Math. Soc. Transl. 5 (1957) pp. 1-33.

[11] -»- On some non linear extremal problems for bounded analytic functions, Dokl. Akad. Nauk SSSR 92 (1953) pp. 243-245. (Russian) MR 15-515.

[12] Herns, M.: A lemma on positive harmonic functions, Ann. of Math. 52 (1950) pp. $568-573$.

[13] - - The problem of Milloux for functions analytic throughout the interior of the unit circle. Amer. J. Math. 67 (1945) pp. 212-234.

[14] -"- Hardy Classes on Riemann Surfaces, Springer-Verlag Lecture Notes No. 98, Berlin, 1969.

[15] Hejhal, D. A.: Linear extremal problems for analytic functions, Acta Math. 128 (1972) pp. 91-122.

[16] -》- Theta functions, kernel functions, and Abelian integrals, Mem. Amer. Math. Soc. 129 (1972) 112 pp.

[17] - - Sur les problèmes linéaires extrémaux pour les fonctions analytiques, C. R. Acad. Sci. Paris 275 (1972) pp. 329-332.

[18] Hewitt, E. and Stromberg, K.: Real and Abstract Analysis, Springer-Verlag, New York, 1965.

[19] LAX, P.: Reciprocal extremal problems in function theory, Comm. Pure Appl. Math. 8 (1955) pp. $437-453$. 
[20] Nevanlinna, R.: Analytic Functions, Springer-Verlag, Berlin, 1970.

[21] -»- Über beschränkte analytische Funktionen, Ann. Acad. Sci. Fenn. Ser. A 32 (1929) no. 7, 75 pp.

[22] Priwalow, I. I.: Randeigenschaften Analytischer Funktionen, VEB Deutscher Verlag der Wissenschaften, Berlin, 1956.

[23] Royden, H.: The boundary values of analytic and harmonic functions, Math. Z. 78 (1962) pp. $1-24$.

[24] WaLSh, J. L.: Interpolation and Approximation, Amer. Math. Soc. Colloquium Publications 1956. 